\title{
Active Laser Guide Star refocusing system for EAGLE instrument
}

Emmanuel Hugot ${ }^{1, \text { a }}$, Fabrice Madec ${ }^{1}$, Sébastien Vives ${ }^{1}$, Elodie Chardin ${ }^{1}$, Marc Ferrari ${ }^{1}$, David Le Mignant $^{1}$, Jean Luc Gimenez ${ }^{1}$, Silvio Mazzanti ${ }^{1}$, Pascal Vola ${ }^{1}$, and Jean Gabriel Cuby ${ }^{1}$

Laboratoire d'Astrophysique de Marseille (LAM), 38 rue F. Joliot Curie, 13388 Marseille Cedex 13, France

\begin{abstract}
We detail the study of a laser guide star (LGS) refocusing system based on an variable curvature mirror (VCM) of high dynamic, in the frame of the EAGLE instrument for the E-ELT. From the top level requirements, an on axis optical design based on an active component is optimised to ensure maximal performance in terms of encircled energy. The refocusing is operated by the VCM, which shape varies with the distance of the sodium layer to the telescope. The VCM system concept is based on an embedded metrology. We detail the finite element analysis (FEA) of the VCM, allowing an optimization of the thickness profile to get an optical quality better than $\lambda / 5$ RMS at each curvature. Mechanical design and manufacturing of prototypes are also presented.
\end{abstract}

\section{Introduction}

For LGS assisted AO systems, the distance from the LGS spot to the telescope pupil ranges from about 80 to $200 \mathrm{~km}$, depending on the Sodium layer altitude and the elevation of the telescope. This variation leads to a defocusing effect on the LGS wave-front sensor which needs to be compensated. In the specific case of the EAGLE multi-object spectrograph [1,2], one of the E-ELT instruments, we propose a solution based on an original optical design including an active optics component. This design could also be applied to other system as for instance ATLAS, the Laser Tomography module for E-ELT [3]. This LGS Variable Curvature Mirror (LGS-VCM) is a $120 \mathrm{~mm}$ spherical active mirror able to achieve a $820 \mu \mathrm{m}$ deflection sag with an optical quality better than $\lambda / 5 \mathrm{RMS}$, allowing the radius of curvature variation corresponding to the required range. Results of FEA simulations, embedded metrology concept and similar mirror prototypes are presented.

\section{Top Level Requirements}

As a baseline, the following specifications are considered (see Tab.1): the laser guide star refocusing system must ensure $100 \%$ of encircled energy in $0.2 \mathrm{arcsec}$, for a $15 \mathrm{arcsec}$ field of view if the launcher is from the telescope side (10arcsec is the launcher is behind M2). The distance from the telescope to the sodium layer varies between $85 \mathrm{~km}$ at zenith and $200 \mathrm{~km}$ for a 60 degrees of inclination.

Table 1. Top Level Requirements and performances

\begin{tabular}{|c|c|c|c|}
\hline Wavelength & Field of view & Altitude of the Na layer & Distance of the Na layer \\
\hline \multirow[t]{3}{*}{$589 \mathrm{~nm}$} & $15 \operatorname{arcsec}$ & $90-100 \mathrm{~km}$ & $85 \mathrm{~km}$ (zenith) - 200km(60deg) \\
\hline & Encircled Energy (100\%) & Pupil quality/stability & \\
\hline & $<0.2 \operatorname{arcsec}$ (diameter) & TBD & \\
\hline
\end{tabular}

\footnotetext{
a e-mail: emmanuel.hugot@oamp.fr
} 


\section{On-axis optical design}

The central obscuration of the telescope allows to propose an on-axis design (Fig.1 left), minimizing optical aberrations. The telescope pupil is re-imaged onto the VCM thanks to a front aspherical lens. The convex VCM produces a divergent beam allowing to tune the size of the central obscuration as needed. After the VCM, a sort of Schmidt telescope produces the intermediate focus where field masks can be located to limit fratricide effects. Performances in terms of Encircled Energy (EE) are plotted on Fig.?? right and summarized in Tab.2. this design focuses $100 \%$ of EE in 0.04 arsec, with a pupil ellipticity of $\pm 0.015 \%$ and a pupil shearing of $0.6 \%$, perfectly inside the specifications of Tab.1. From this design, the range of curvature variation is $\left[3000^{-1}, 1200^{-1}\right]$ over a $120 \mathrm{~mm}$ diameter clear aperture. These values corresponds to a sag of $820 \mu \mathrm{m}$ on the VCM. The main challenge here is to produce such a large sag with a good optical quality.

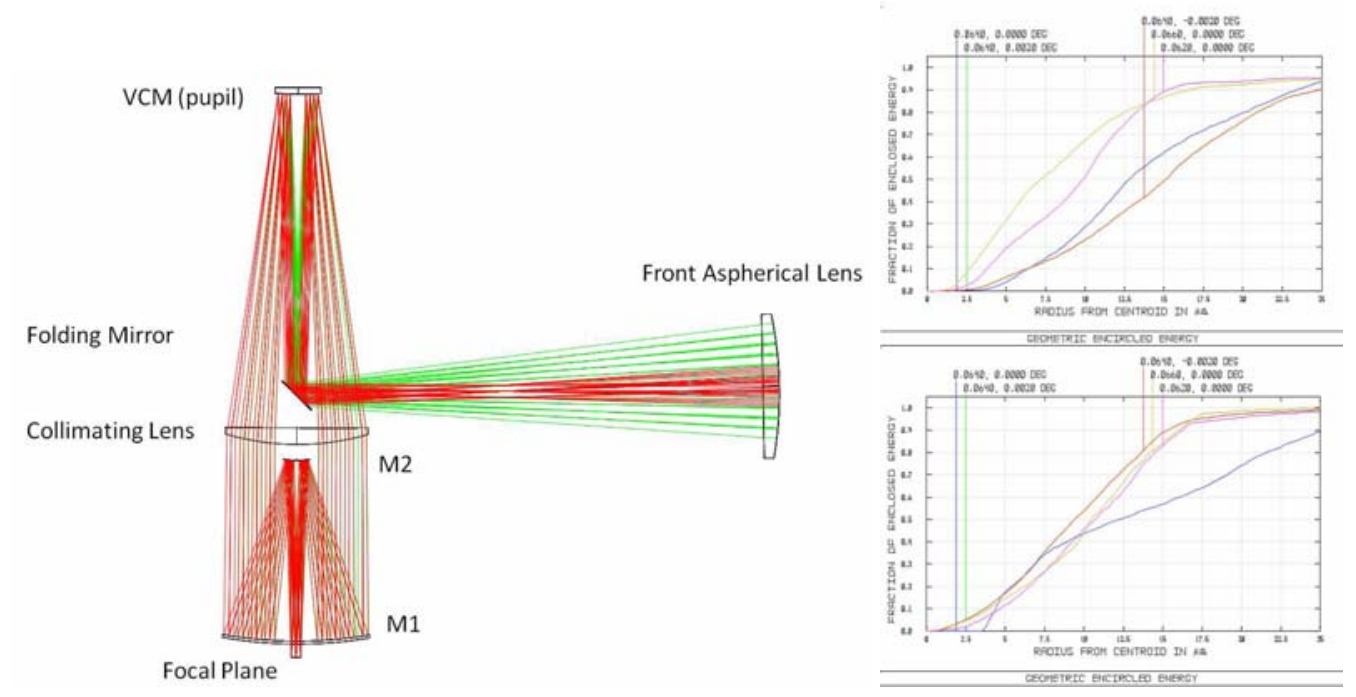

Fig. 1. Left: LGS refocusing - On-axis optical design. Green rays are corresponding to a distance of $80 \mathrm{~km}$, red rays to a distance of $200 \mathrm{~km}$. Right: Encircled energy for the two extreme altitudes: $85 \mathrm{~km}$ (Up) and 200km (Down) within the $0.02 \operatorname{arcsec}(\sim 50 \mu \mathrm{m})$

Table 2. On-axis optical design performances

\begin{tabular}{ccc}
\hline Encircled Energy (100\%) & Pupil ellipticity & Pupil shearing \\
\hline $0.04 \operatorname{arcsec}$ & $\pm 0.015 \%$ & $0.6 \%(70 \mu \mathrm{m})$ \\
\hline
\end{tabular}

\section{VCM-LGS system concept}

The global concept for such a high dynamic VCM, drawn on Fig.2 is the following. A pressure is applied on the rear face of a thin metallic meniscus, bending it into a spherical shape. The goal was to design a mirror able to produce more than $800 \mu \mathrm{m}$ of deflection for a pressure lower than 8 bars, which is the pressure used for the existing VCM for the VLTI [4]. In order to guarantee the exact radius 
of curvature, the system will use an embedded metrology measuring the sag of the mirror to directly calculate the curvature. A sensor is used to measure the displacement of the back side of the mirror. The specifications of the sensor, derived from the optical specification, are given in Tab.3. The system has an embedded electronic and a remote control for the pressure through an FPGA. The pressure is adjusted through valves regulating gas flow in two steps: fast regulation at \pm 1 bar and regulated flow at $1 \mathrm{mbar}$. The gas input and work station are placed far from the system, allowing a compact and lightweight system.

Table 3. Sensor specifications for the embedded metrology

\begin{tabular}{ccc}
\hline Parameter & Value & Remark \\
\hline Stroke & $1.3 \mathrm{~mm}$ & Sag measurement \\
Accuracy & $\pm 0.1 \mu \mathrm{m}$ & Stability \\
Pressure & 0 to $8 \mathrm{bar}$ & \\
Bandwidth & $1 \mathrm{~Hz}$ & Not critical \\
\hline
\end{tabular}

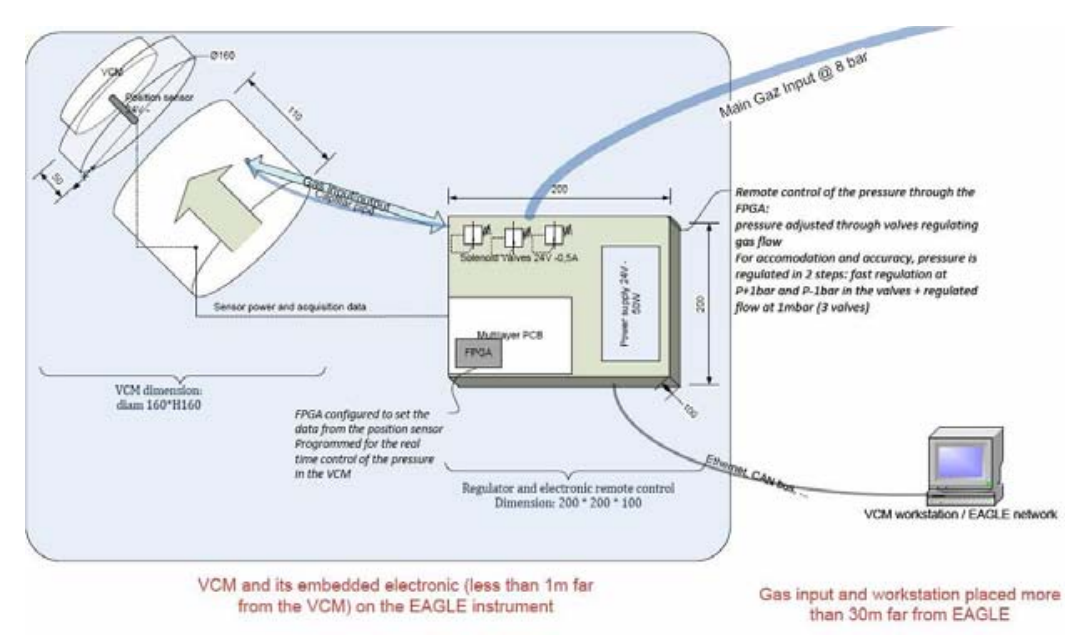

Fig. 2. VCM-LGS system concept

\section{VCM Finite Element Analysis}

The principle of VCMs is based on elasticity theory of circular plates under load [5]. The analytical study allows defining a specific geometry for the mirror, in order to bend it spherically under a specific load. In our case, the convex optical surface is made of a thin meniscus with a variable thickness distribution (VTD) (Fig.3 left), linked to a outer rigid ring with a very thin collar. The VCM will be made in thermal treated AISI 420. An air pressure is applied on the rear face of the meniscus, bending it to change its radius of curvature.

FEA is performed using Marc/Mentat software [6]. The axisymmetric model is made of 43,000 quadrangles, 44,000 nodes, and the sampling on the optical radius is of 500 points. The first results shows that a geometrical non linearity appears when the pressure increases (Fig.3 Right), and the residual error shape varies (Fig.4 Left). To compensate for this variation, the VTD is optimized by 
an iterative method, in order to keep an optical quality better than $\lambda / 5$ RMS over the full range of curvature (Fig.4 right) [7].
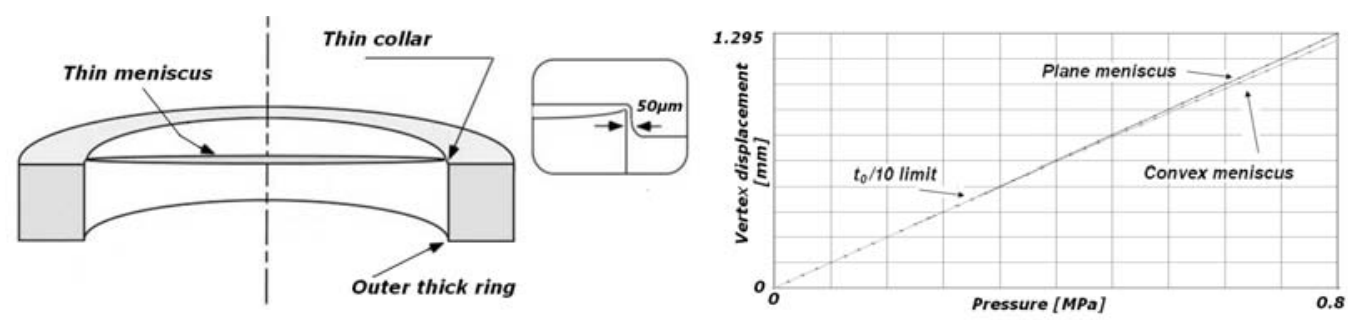

Fig. 3. Left: VCM profile and zoom on the collar. Right: Non linearity of the vertex displacement
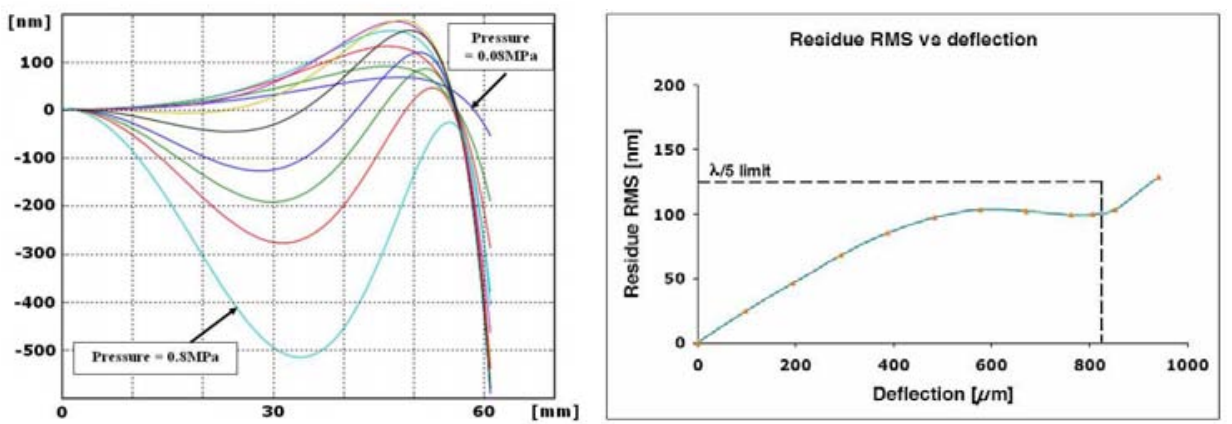

Fig. 4. Left: Change of the residue shape with the pressure. Right: RMS residue versus sag on the clear aperture.

\section{Mechanical design and manufacturing}

The mechanical design of the mounting ensure the sealing of the pressure chamber and the centering of the mirror and the sensor (Fig.5 Left). Three prototypes are manufactured and are under polishing. Two of them will be used for the optical characterization and the third one is dedicated to the ageing tests, corresponding to 10 years of operation. The first prototype is now polished with an optical quality of $\lambda / 30$ RMS over the $120 \mathrm{~mm}$ clear aperture (Fig.5 Right).

\section{Conclusion}

We design an active optics refocusing system for laser guide stars, giving optimised optical performances in the frame of EAGLE instrument for the E-ELT. The active component is a variable curvature mirror, able to compensate for the distance variation between the sodium layer and the telescope during observations. This metallic VCM can be bend under pressure, producing a sag of $1 \mathrm{~mm}$ with an optical quality of $\lambda / 5$ RMS over a $120 \mathrm{~mm}$ clear aperture. Three prototypes have been manufactured for the experimental validation. Metrology, tests and comparison with FEA are under going at time of writing. 

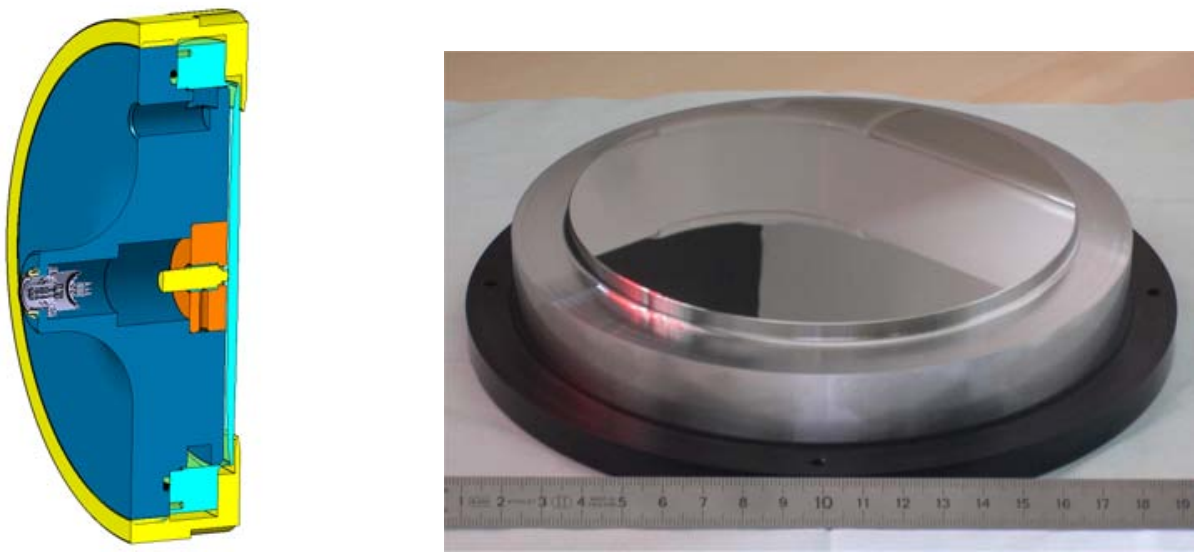

Fig. 5. Left: Housing. Right: First prototype of convex VCM after polishing.

\section{Acknowledgments}

This work was supported by the French Agence Nationale de la Recherche (ANR) program 06BLAN-0191 and The European Community (Framework Programme 7, E-ELT Preparation, contract No INFRA-2.2.1.28).

\section{References}

1. J.G.Cuby et al, SPIE Proc. on Astronomical Adaptive Optics Systems and Applications IV, 7439B, 7439B-18 (2009).

2. J.G.Cuby et al, SPIE Proc. on Astronomical Telescopes and Instrumentation, 7014, 70141K (2008).

3. T.Fusco et al, this conference.

4. M.Ferrari, Astron. Astrophys., Suppl.Ser.128, (1998) 221-227.

5. S.P.Timoshenko, S.Woinovsky-Krieger, Theory of plates and shells, (McGraw-Hill Co. 1959).

6. Marc/Mentat, MSC software corporation.

7. E.Hugot, M.Ferrari, G.R.Lemaitre, F.Madec, S.Vives, E.Chardin, D.Le Mignant, J.G.Cuby, Optic Letters, (submitted). 